\title{
Reflexiones de estudiantes de profesorado de Física y Química sobre Naturaleza de la Tecnología en el contexto de la controversia Tesla-Edison
}

\author{
Antonio García-Carmona \\ Universidad de Sevilla (US), España
}

Resumen. Una comprensión informada de la Naturaleza de la Tecnología debería formar parte de la alfabetización científica básica de la ciudadanía. Esto implica que, más allá de los propósitos educativos propios del área curricular de Tecnología, la educación científica tendría que plantear también entre sus objetivos conocer los rasgos más característicos de la Tecnología como sistema, incluyendo sus relaciones con la Ciencia. Conscientes de ello, se llevó a cabo una experiencia educativa para promover la comprensión de nociones básicas de Naturaleza de la Tecnología en la formación inicial del profesorado de Ciencia. Para tal propósito, se diseñó una actividad consistente en la lectura de la controversia histórica entre Tesla y Edison sobre la corriente eléctrica, y la reflexión sobre algunas cuestiones de Naturaleza de la Tecnología visibles en esta. Participaron en el estudio 16 estudiantes de profesorado de la especialidad de Física y Química de Educación Secundaria, que conformaban un grupo-clase natural. Los resultados revelan una progresión moderada de la comprensión de los estudiantes sobre los aspectos tratados, si bien suponen una novedad importante en esta línea de investigación. Se finaliza con las limitaciones e implicaciones del estudio realizado.

Palabras clave: alfabetización científica; formación inicial del profesorado; historia de la tecnología; naturaleza de la tecnología

Reflexões dos estudantes de Licenciatura em Física e Química sobre a Natureza da Tecnologia no contexto da controvérsia Tesla vs. Edison

Resumo. Uma compreensão informada da Natureza da Tecnologia deve fazer parte da alfabetização científica básica dos cidadãos. Isto implica que, para além dos propósitos educacionais da área curricular da Tecnologia, a educação científica também deve contemplar entre os seus objetivos o conhecimento dos aspectos mais característicos da Tecnologia como um sistema, incluindo as relações com a Ciência. Conscientes disso, realizou-se uma experiência pedagógica para promover a compreensão das noções básicas sobre a Natureza da Tecnologia na formação inicial dos professores de Ciências. Para este fim, criou-se uma atividade que consiste na leitura da batalha histórica entre Tesla e Edison sobre corrente elétrica, e na reflexão sobre algumas das questões da Natureza da Tecnologia visiveis nesta controvérsia. Participaram do estudo 16 alunos de Licenciatura em Física e Química. Os resultados revelam uma progressão moderada na compreensão dos aspectos tratados pelos estudantes, embora representem uma importante novidade nesta linha de pesquisa. Conclui com as limitações e implicações do estudo realizado.

Palavras-chave: alfabetização científica; formação inicial de professores; história da tecnologia; natureza da tecnologia.

Physics and Chemistry teacher students' reflections about Nature of Technology in the context of the Tesla-Edison controversy

Abstract. An informed understanding of the Nature of Technology should be part of the basic scientific literacy of citizenship. This suggests that, beyond the own educational goals of the curricular area of Technology, science education would also have to set goals aimed at knowing the more characteristic features of Technology as a system, including its relationships with science. Consequently, an educational experience was conducted in order to promote the understanding of basic notions on Nature of Technology in initial science teacher education. To this end, an activity was designed consisting of reading the historical controversy between Tesla and Edison about electric current, and reflecting on some Nature of Technology questions, which are visible in this. A natural class-group of 16 Physics and Chemistry teacher students of Secondary Education participated in the study. The results reveal the students experimented a moderate progression in their understandings on the aspects of Nature of Technology addressed. Even so, the study constitutes an important novelty in this line of research. The limitations and implications of the study are commented finally.

Keywords: History of Technology; Nature of Technology; Scientific literacy; Initial teacher education 


\section{Introducción}

Desde hace años se viene reivindicando la comprensión de nociones básicas sobre Tecnología como parte esencial de una adecuada alfabetización científica para la ciudadanía (Acevedo-Díaz, 1998; Cajas, 2001; Criado y García-Carmona, 2011; Maiztegui et al., 2002). Esto no solo debe traducirse en disponer de un área curricular específica de Tecnología, que ya existe. Se trata también de establecer conexiones entre la Ciencia y la Tecnología para enriquecer, en este caso, la educación científica. Como Maiztegui et al. (2002) sostienen, "la inclusión de la Tecnología en la educación científica constituye un paso más, imprescindible, hacia la conformación de unas nuevas humanidades que incorporen saberes científicos y tecnológicos como parte sustancial de la cultura" (p. 149).

Más recientemente, en los estándares nacionales para la enseñanza de la Ciencia en Estados Unidos (National Research Council, 2012), se dice que:

(...) una educación científica que se concentre predominantemente en los productos del trabajo científico (...) que ignore las muchas aplicaciones importantes de la Ciencia en el mundo, tergiversa la Ciencia y margina la importancia de la Ingeniería. ${ }^{1}(\ldots)$ los estudiantes deben aprender cómo se utiliza la Ciencia, en particular a través del proceso de diseño ingenieril, y deben llegar a apreciar las distinciones y relaciones entre Ingeniería, Tecnología y aplicaciones de la Ciencia. (p. 201; traducción propia)

Asimismo, en el marco de PISA, el concepto de competencia científica integra un conocimiento básico sobre las diferencias y relaciones entre Ciencia y Tecnología (Organización para la Cooperación y el Desarrollo Económicos [OCDE], 2017, p. 93). De igual modo, en el preámbulo del currículo de Física y Química para la Educación Secundaria Obligatoria (Ministerio de Educación, 2015), se habla explícitamente de la necesidad de promover aprendizajes contextualizados en las relaciones entre la Ciencia, la Tecnología y la Sociedad (CTS).

Aun así, como ya denunciaran Maiztegui et al. (2002) hace casi 20 años, la Tecnología sigue siendo una "dimensión olvidada" en la educación científica que habitualmente se desarrolla en las aulas. O bien, malinterpretada en movimientos educativos recientes como STEM, en los que la Tecnología se suele identificar simplemente con el uso de herramientas TIC en los procesos de enseñanza/aprendizaje de la Ciencia (García-Carmona, 2020). Por tanto, se hace necesario que el profesorado de Ciencia adquiera una conceptualización apropiada del significado de Tecnología; o lo que es igual, una comprensión informada de la Naturaleza de la Tecnología (NDT) (AcevedoDíaz y García-Carmona, 2016; Waight y Abd-El-Khalick, 2012).

Las pocas investigaciones realizadas sobre concepciones de NDT con estudiantes (e.g., DiGironimo, 2011; García-Carmona, 2006), con profesorado (e.g., AcevedoDíaz, Vázquez-Alonso, Manassero-Mas y Acevedo-Romero, 2005; Ferreira-Gauchía, Vilches y Gil-Pérez, 2011) o con ambos colectivos conjuntamente (e.g. , Acevedo-Díaz, Vázquez-Alonso, Manassero-Mas y Acevedo-Romero, 2003), revelan que estos tienen, en general, una imagen empobrecida o poco informada sobre la Tecnología. Por ejemplo, se suele asimilar la Tecnología como "Ciencia aplicada"; que la existencia de

${ }^{1}$ En este trabajo, la Ingeniería se concibe como la parte de la Tecnología dedicada a la construcción de artefactos (Raynaud, 2018). 
la Tecnología es una consecuencia de la Ciencia, o que la Tecnología tiene un menor estatus que la Ciencia por ser considerada una "actividad práctica" frente al "desempeño intelectual" de la Ciencia. También es frecuente que se identifique la Tecnología solo con los instrumentos, o con los avances tecnológicos modernos, obviando así su desarrollo histórico y complejidad.

Aparte de esos estudios diagnósticos, no conocemos trabajos orientados específicamente a mejorar la comprensión de aspectos básicos de NDT desde la educación científica. Por tanto, conscientes de esta necesidad y de que, quizá, un primer paso para mejorar tal situación de la NDT en la educación científica sea impulsarla en la formación inicial del profesorado de Ciencia, decidimos llevar a cabo el presente estudio. Se trata de una investigación para evaluar la eficacia de una experiencia educativa enfocada a promover algunos aprendizajes sobre NDT; en este caso, con estudiantes de profesorado de Física y Química de Educación Secundaria (en adelante, EPFQ). Se utiliza como contexto, para ello, un relato de Historia de la Tecnología potencialmente útil para reflexionar sobre NDT: la controversia entre Tesla y Edison sobre la corriente eléctrica (Acevedo-Díaz y García-Carmona, 2016).

Las cuestiones de investigación en las que se concreta el estudio son las siguientes:

1. ¿Qué concepciones iniciales muestran los EPFQ sobre los componentes característicos de la Tecnología como sistema, así como sobre las similitudes y diferencias entre Ciencia y Tecnología, tras una primera lectura de la controversia?

2. ¿Qué progresiones se observan tras la puesta en común y discusión de esas concepciones iniciales de los EPFQ sobre los aspectos de NDT tratados?

\section{Marco teórico}

La Tecnología es difícil de definir al tratarse de una disciplina del saber compleja y multifacética (Raynaud, 2018). Para Kline (1985), la Tecnología puede referirse a: (i) el conjunto de productos artificiales fabricados por las personas (herramientas, instrumentos, máquinas, artefactos y todo tipo de sistemas); (ii) los conocimientos técnicos, metodologías, capacidades y destrezas necesarias para poder diseñar y realizar las tareas productivas (know-how); (iii) los recursos humanos y materiales del sistema sociotécnico de producción; y (iv) el sistema sociotécnico necesario para el uso y mantenimiento de los productos fabricados, que incluye también los aspectos legales.

De las perspectivas anteriores, las dos primeras suelen ser las más reconocidas, pero las dos últimas, que tienen que ver con la dimensión organizativa y el entramado social de la Tecnología, suelen ser poco identificadas. Si bien, una conceptualización más actualizada de la NDT trata de promover una visión de la Tecnología holística; esto es, que, junto a explicar sus fundamentos epistemológicos y ontológicos, deberían atenderse también los rasgos del trabajo de las comunidades de tecnólogos como grupos sociales y las interacciones CTS (Acevedo-Díaz y García-Carmona, 2016).

En esta línea, Waight y Abd-El-Khalick (2012) apuntan que la Tecnología puede ser caracterizada por un proceso complejo que: (i) ejemplifica el artefacto, la actividad asociada al mismo y el proceso que lo genera; (ii) resalta la complejidad del diseño, 
el desarrollo y la implementación de la innovación; (iii) ilustra las interacciones entre la Tecnología y los ámbitos políticos, económicos y culturales; (iv) revela los beneficios y obstáculos intencionales y no intencionales; y, lo más importante, (v) interroga críticamente las complejidades de la innovación y cómo estas se manifiestan en diferentes contextos.

Además de ello, Acevedo-Díaz y García-Carmona (2016) añaden que, de la misma forma que sucede con las comunidades de científicos, las comunidades de tecnólogos tienen sus propios ámbitos de problemas, teorías, métodos, procedimientos y técnicas. Los tecnólogos adaptan conocimiento científico a su peculiar modo de hacer, junto a otros conocimientos tácitos de carácter técnico, así como tradiciones basadas en la experiencia acumulada y prácticas empresariales.

Es importante señalar también que, aunque la Ciencia y la Tecnología son ontológicamente diferentes, sus influencias mutuas son palpables (Acevedo-Díaz, 2006). La Tecnología se ha ido haciendo cada vez 'más científica' porque ha ido incorporando a su quehacer conocimientos provistos por la Ciencia y metodologías de investigación y desarrollo más sistemáticas. Una perspectiva que, obviamente, no debe llevar a identificar ingenuamente Tecnología con "Ciencia aplicada" (Maiztegui et al. 2002; Valdés, Valdés, Guisasola y Santos, 2002). De manera similar, la Tecnología juega un papel esencial en el desarrollo científico; y no solo porque propicia instrumentos para la investigación científica, sino porque, en muchos casos, permite también "generar fenómenos" para su estudio en Ciencia. Como señalan Valdés, Valdés, Guisasola y Santos (2002),

(...) el primer circuito eléctrico construido por Volta, la desviación de una aguja magnética debido a una corriente eléctrica (experimento de Oersted) y el fenómeno de la inducción electromagnética (experimentos de Faraday), bases sobre las cuales se erigió el electromagnetismo, resultaron fenómenos que, más que estudiados por medio de determinada instrumentación [tecnológica], fueron "originados mediante ella», y, por tanto, correspondieron a "un "mundo" creado por el hombre». (p. 116; corchetes añadidos)

Asimismo, tanto la Ciencia como la Tecnología comparten numerosos problemas de investigación en los que, con frecuencia, las dos disciplinas suelen alcanzar estados de hibridación tales que resulta difícil distinguir cuál es el papel o aportación de cada una. En estos casos suele hablarse de Tecnociencia (Echevarría, 2005).

Un buen modo de introducir nociones de NDT en las clases de Ciencia y Tecnología es mediante el análisis reflexivo de algunos pasajes de la Historia de la Tecnología (Acevedo-Díaz, 2010; Acevedo-Díaz y García-Carmona, 2016). Este recurso permite contextualizar de forma explícita algunos aspectos de NDT; por ejemplo, aquellos referidos a la manera en que los ingenieros y tecnólogos afrontan su actividad. Asimismo, puede ser útil para ilustrar diversas cuestiones epistemológicas, ontológicas, axiológicas y sociológicas vinculadas a la Tecnología, situando así el contenido en un contexto humano, social y cultural. 


\section{Metodología}

\subsection{Participantes}

La actividad se implementó con 16 EPFQ (6 hombres y 10 mujeres; rango de edades = 22-32 años; promedio = 25.9 años; SD = 3.1 años), con titulaciones universitarias en Biotecnología (1), Ciencias Físicas (3), Ciencias Químicas (9), Ingeniería (1) y Ciencias Ambientales (2). Los EPFQ cursaban la asignatura de Aprendizaje y Enseñanza de las materias de Física y Química (6 créditos), correspondiente al Máster de Profesorado en Educación Secundaria, impartido por la Universidad de Sevilla. Se trababa de un grupo-clase natural al que se tuvo acceso en el momento de llevar a cabo la experiencia educativa (muestra de conveniencia). Para la implementación de la actividad, los EPFQ se organizaron en grupos pequeños de trabajo de 2 o 3 miembros, hasta conformar un total de 6 grupos $(\mathrm{G} 1, \ldots, \mathrm{G} 6)$.

\subsection{Descripción de la actividad y su implementación}

La actividad consistió en la lectura reflexiva de un relato sobre la controversia histórica entre Tesla y Edison sobre la corriente eléctrica, a fin de discutir sobre algunas cuestiones de NDT. El relato se expone completamente en otro trabajo (Acevedo y García-Carmona, 2016). En síntesis, el relato (i) permite reflexionar sobre el papel de los agentes sociales, individuales y colectivos en el desarrollo de la controversia; (ii) ayuda a dar una visión de la Tecnología como un sistema complejo que incluye numerosos componentes característicos, que van desde los instrumentos, recursos, métodos, organización, etc. hasta los estéticos, sociales, políticos, económicos, ambientales, etc.; (iii) favorece una discusión acerca de las relaciones entre Ciencia y Tecnología; y (iv) pone de relieve los valores de la Tecnología (racionalidad técnica, beneficio económico, etc.).

La actividad fue implementada en tres fases:

- Fase I: Lectura de la controversia y respuestas a las cuestiones. Sin instrucción previa, los EPFQ respondieron en grupo a las cuestiones de la tabla $1 .^{2}$ El formador del profesorado animó a cada grupo para que sus respuestas fueran resultado de una discusión y consenso entre todos los miembros. Los grupos debían registrar sus respuestas iniciales en un informe. Esta primera fase, que se realizó en una sesión de clase de 2 horas, permitió diagnosticar las ideas iniciales de los futuros docentes sobre los aspectos de NDT tratados.

Tabla 1. Cuestiones para la reflexión sobre NDT en el contexto de la controversia Tesla-Edison.

1. De acuerdo con lo que has leído, ¿qué componentes crees que caracterizan mejor a la Tecnología como sistema? Intenta elaborar con ellos una definición de Tecnología.

2. Tras la lectura de la controversia, ¿qué similitudes y diferencias establecerías entre la Ciencia y la Tecnología?

Fuente: elaboración propia.

${ }^{2}$ Estas dos cuestiones forman parte de un conjunto más amplio de preguntas para reflexionar sobre NDT, planteadas en Acevedo-Díaz y García-Carmona (2016). 
- Fase II: Discusión en clase de las respuestas iniciales de los grupos a las cuestiones. Tras responder a las cuestiones planteadas, los grupos compartieron y discutieron sus opiniones en una sesión de clase de 1.5 horas aproximadamente. El formador moderóla discusión entre los grupos e introdujo aclaraciones, explicaciones, cuestiones adicionales, etc. para enriquecerla todo lo posible. Cuando los EPFQ exponían ideas poco informadas sobre NDT, el formador procuraba crear un conflicto cognitivo para que reconsideraran sus argumentos iniciales. La intención era que los grupos llegaran a conclusiones comunes sobre los distintos aspectos de NDT abordados, aunque sin adoctrinamientos.

- Fase III: Conclusiones de los grupos tras la sesión de puesta en común. Después de la discusión entre los grupos sobre las cuestiones de NDT formuladas, cada grupo debía revisar sus respuestas iniciales tratando de mejorar sus argumentos originales. Para ello contaron con una semana de plazo. Las respuestas finales, surgidas de esta última revisión, fueron registradas por los grupos en sus informes, junto con las respuestas iniciales.

\subsection{Análisis de la información}

Las respuestas de los grupos a las cuestiones de NDT se analizaron mediante la rúbrica de la tabla 2. Esta fue elaborada por el autor con la colaboración de un colega investigador, a partir de un análisis preliminar e inductivo de las respuestas de los grupos (Mayring, 2000), y de acuerdo con el marco teórico expuesto. La rúbrica consta de 5 niveles de progresión (niveles de 0 a 4) para cada aspecto de NDT analizado. El nivel máximo (nivel 4) corresponde a las respuestas más completas, de acuerdo con el nivel deseable para una comprensión básica de los aspectos de NDT aludidos en la narración del caso. La categorización de las respuestas va decreciendo de nivel, según lo incompletas que estas sean, hasta llegar al nivel más bajo (nivel 0), donde se encuentran las inadecuadas o que no se refieren a ninguno de los rasgos indicados en el nivel 4.

Tabla 2. Rúbrica de evaluación de las respuestas.

1. De acuerdo con lo que has leído, ¿qué componentes crees que caracterizan mejor a la Tecnología como sistema? Intenta elaborar con ellos una definición de Tecnología.

Niveles de respuestas

- Nivel 4 (máximo): Se define la Tecnología como un sistema complejo, orientado a resolver ciertos problemas, que integra componentes de diversa naturaleza, citando, al menos, 6 de los siguientes:

(i) Instrumentos y artefactos técnicos.

(ii) Recursos naturales y artificiales.

(iii) Destrezas y habilidades técnicas (know-how).

(iv) Conocimientos científicos.

(v) Procesos de producción (empresas industriales).

(vi) Financiación (entidades bancarias).

(vii) Control y mantenimiento (servicios públicos).

(viii)Valores (e.g., sostenibilidad, bienestar) y acuerdos sociales.

(ix) Decisiones políticas, legales y administrativas (e.g., permisos de concesióny leyes reguladoras).

(x) Preferencias culturales y estéticas.

(xi) Innovación. 
- Nivel 3: Se define la Tecnología de manera similar a la del nivel 4, citando 4 o 5 de los componentes indicados en el nivel 4.

- Nivel 2: Se define la Tecnología de manera similar a la del nivel 3, citando 3 de los componentes indicados en el nivel 4.

- Nivel 1: Se definen la Tecnología de manera similar a la del nivel 3, citando 1 o 2 de los componentes indicados en el nivel 4.

- Nivel 0: No se define Tecnología ni se cita ninguno de los componentes indicados en el nivel 4.

2. Tras la lectura de la controversia, ¿qué similitudes y diferencias establecerías entre la Ciencia y la Tecnología?

Niveles de respuesta

- Nivel 4 (máximo): Se identifican, al menos, 3 similitudes y 3 diferencias de entre las que se indican a continuación:

Similitudes:

(i) Tanto la Ciencia como la Tecnología están en permanente desarrollo.

(ii) La Ciencia y la Tecnología emplean métodos sistemáticos en su construcción y desarrollo.

(iii) La creatividad y la imaginación juegan un papel esencial en el desarrollo de la Ciencia y de la Tecnología.

(iv) Tanto la Ciencia como la Tecnología generan conocimientos sobre aspectos mensurables, que se validan empíricamente.

(v) La Ciencia y la Tecnología abordan muchos problemas comunes, siendo la contribución de ambas difícil de diferenciar en estos casos (e.g., Tecnociencia).

(vi) La Ciencia y la Tecnología requieren de apoyo político y financiero en su desarrollo.

Diferencias:

(i) La Ciencia busca explicar fenómenos de la naturaleza, mientras que la Tecnología trata de elaborar productos (máquinas, artefactos, aplicaciones, etc.) que resuelvan problemas, o bien que satisfagan o creen necesidades sociales.

(ii) El conocimiento tecnológico tiene una finalidad práctica, mientras que el conocimiento científico no necesariamente.

(iii) El conocimiento científico se caracteriza por tener una estructura lógico-formal, mientras que el tecnológico incluye también aspectos prácticos no necesariamente regidos por una estructura lógica.

(iv) En el desarrollo tecnológico se emplea a menudo una metodología de ensayo y error para depurar el producto final. Esto no suele suceder en la Ciencia.

(v) El desarrollo científico suele presentarse sobre todo en publicaciones (artículos, libros, etc.), y el desarrollo tecnológico en patentes de modo preferente.

- Nivel 3: Se identifican 2 similitudes y 2 diferencias de las indicadas en el nivel 4.

- Nivel 2: Se identifican, 1 similitud y 1 diferencia de las indicadas en el nivel 4.

- Nivel 1: Se identifican solo similitudes o solo diferencias, de las indicadas en el nivel 4, pero no ambas a la vez.

- Nivel 0: No se identifican similitudes o diferencias, de las indicadas en el nivel 4, o bien estas son inadecuadas.

Fuente: elaboración propia.

Una vez elaborada la rúbrica, la categorización de las respuestas de los grupos se hizo combinando métodos de análisis inter-e intra-evaluadores de manera iterativa. El autor y su colega analizaron las respuestas por separado para luego confrontar sus categorizaciones. En esta primera fase del análisis, los investigadores mostraron coincidencias en la categorización de 17 de las 24 respuestas. Los casos en los que se encontraron discrepancias fueron sometidos de nuevo a revisión y discusión por 
parte de ambos investigadores. Fruto de ello se logró la categorización de 22 de las 24 respuestas. Aproximadamente 3 meses después, el autor volvió a revisar toda la categorización y resolvió la de las dos respuestas pendientes, teniendo en cuenta el marco teórico y lo discutido en torno a ellas con su colega. De este modo se consiguió la categorización definitiva de todas las respuestas de los grupos.

\section{Resultados}

\subsection{Componentes que caracterizan la Tecnología como sistema}

Tras la lectura de la controversia, los grupos mostraron en sus respuestas iniciales un grado de comprensión ubicada entre los niveles 1 y 2 (figura 1). De acuerdo con la rúbrica de evaluación, las respuestas se caracterizaron por una referencia limitada a componentes característicos de la Tecnología como sistema. Los componentes más citados fueron los instrumentos y artefactos técnicos, y los valores y acuerdos sociales ligados a la Tecnología (4 de 6 grupos en ambos casos), seguido de destrezas y habilidades técnicas (3 de 6 grupos), conocimientos científicos (2 de 6 grupos) e innovación (2 de 6 grupos). Por tanto, 6 de los 11 componentes de la Tecnología señalados en la rúbrica (recursos naturales y artificiales, procesos de producción, financiación, etc.) no fueron mencionados. Los que siguen son dos ejemplos de respuestas situadas en los niveles 1 y 2 respectivamente:

G1: La Tecnología se caracteriza por diferentes factores: viabilidad económica y técnica, repercusión social y sostenibilidad. [...] se puede ver [en el relato de la controversia] cómo el desarrollo de la corriente continua era más caro, peligroso y con altas perdidas de energía eléctrica por el efecto Joule. [...]. (Nivel 1; corchetes añadidos)

G3: [La Tecnología] modifica nuestras condiciones de vida, intentando dar respuesta a nuestras necesidades. Dependiendo del uso que se haga de ella, puede ser muy beneficiosa o perjudicial; está en continuo desarrollo, facilita la realización de una actividad, [e] innova y busca ser práctica. [La Tecnología] es el producto de las actividades humanas que buscan facilitar la realización de cualquier labor mediante el desarrollo de artificios. (Nivel 2; corchetes añadidos)

Figura 1. Progresión de las concepciones de los grupos de EPFQ con respecto a los componentes que caracterizan a la Tecnología como sistema.

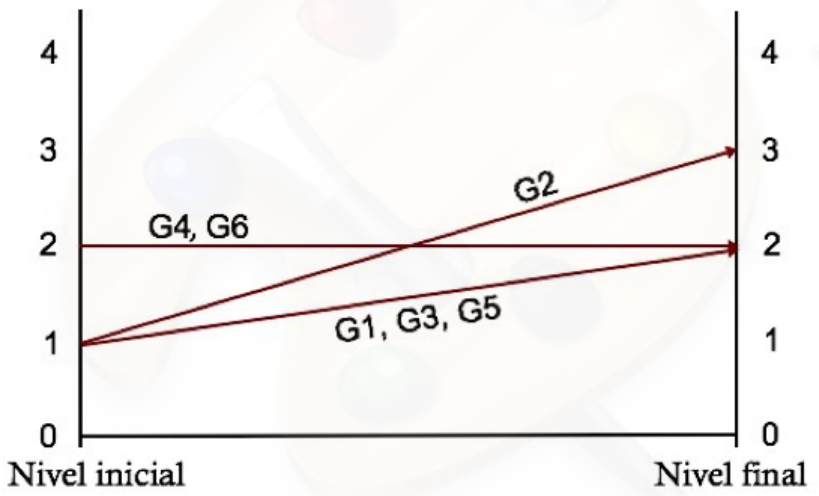

Fuente: elaboración propia. 
Durante la puesta en común, los grupos discutieron sus respuestas. El papel del formador de los EPFQ se limitó a animar el debate, moderarlo y reconducirlo cuando fue necesario; si bien, no trató de corregir ni matizar ninguna de las respuestas o posiciones expuestas, a fin de que fueran los propios EPFQ quienes repensaran sus respuestas tras la discusión en clase.

Cuatro de los seis grupos mostraron alguna progresión en sus respuestas. No obstante, ninguno alcanzó el nivel 4 (máximo) y solo uno (G2) se situó en el nivel 3. De modo que, en general, la progresión de los niveles de comprensión fue moderada. Los grupos que pasaron al nivel 2 añadieron a sus respuestas componentes que ya fueron citados en las respuestas iniciales de otros grupos, señalados más arriba. Solamente uno de estos grupos (G1) añadió la financiación como factor clave para la Tecnología.

El grupo G2 alcanzó el nivel 3 añadiendo a su respuesta inicial alusiones al conocimiento científico, los valores sociales y la innovación:

G2: [...] llegamos a la conclusión de que son tres los componentes de la Tecnología: técnico, económico y social. Actualmente también puede destacarse como importante la sostenibilidad. La Tecnología, a partir de unos conocimientos científicos y tecnológicos, supone la búsqueda de innovación, aplicabilidad y viabilidad técnica y económica, siempre con vistas a la repercusión social que implica todo ello. (Nivel 3)

\subsection{Similitudes y diferencias entre Tecnología y Ciencia}

En cuanto a determinar similitudes y diferencias entre Tecnología y Ciencia, se encuentra que inicialmente los seis grupos se ubican en niveles de comprensión bajos (niveles 1 y 2). Véase la figura 2. Tres grupos se ubican en el nivel 1 al señalar apropiadamente solo similitudes o solo diferencias entre ambas disciplinas, de acuerdo con la rúbrica. Así, el grupo G5 se centra en indicar únicamente diferencias entre Ciencia y Tecnología; y en una de ellas, además, incurre en la concepción errónea de que la Ciencia no está influenciada por asuntos económicos o sociales:

G5: La Ciencia busca el conocimiento de los fenómenos de la naturaleza, [mientras que] la Tecnología aplica el conocimiento para cubrir necesidades (resolver problemas). La Ciencia es "independiente" de lo económico/social, [en cambio] la Tecnología busca el beneficio económico y/o social. La Ciencia difunde su conocimiento mediante publicaciones, [y] la Tecnología lo hace mediante patentes. (Nivel 1; corchetes añadidos)

Mientras que, por ejemplo, el grupo G4 solo señala una similitud entre Ciencia y Tecnología medianamente adecuada (ambas tienen un impacto económico), pero en las diferencias considera que el conocimiento manejado en la Tecnología es solamente práctico: "Con respecto a las diferencias, la Ciencia es un conocimiento básico y teórico, mientras que la Tecnología es un conocimiento práctico. [...]Asimismo, ambas buscan rendimiento económico." (G4; Nivel 1) 
Figura 2. Progresión de las concepciones de los grupos de FPFQ con respecto a las similitudes y diferencias entre Ciencia y Tecnología.

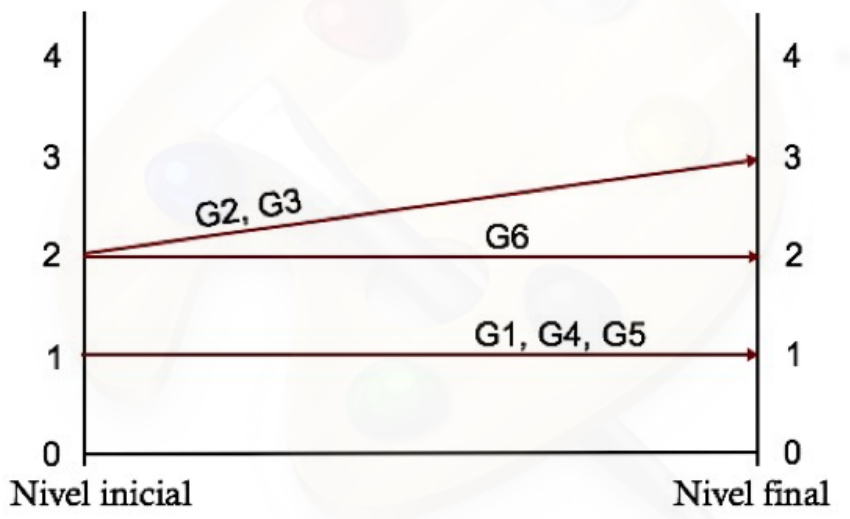

Fuente: elaboración propia.

En el nivel 2, tal y como se recoge en la rúbrica, se ubican los otros tres grupos debido a que sus respuestas iniciales señalan adecuadamente solo una similitud y una diferencia entre Ciencia y Tecnología. Por ejemplo:

G6: La Ciencia tiene como primer objetivo obtener conocimiento, pero no persigue beneficio económico (una rentabilidad). La Tecnología se desarrolla buscando un beneficio económico. Similitudes: Ambas requieren de conocimientos para su desarrollo, sirven como herramientas para resolver problemas de la sociedad, requieren recursos económicos para su desarrollo, y tienen una repercusión social, tanto para bien como para mal. (Nivel 2)

Tras la puesta en común, se obtiene que solo dos de los seis grupos logran progresar a un nivel más alto; en este caso, del nivel 2 al 3 (grupos G2 y G3). Sucede que, si bien la mayoría de los grupos incluyen alguna mejora en sus respuestas, estas solo consisten en añadir alguna similitud o alguna diferencia; lo que, de acuerdo con la rúbrica, no supone un aumento de nivel. Por ejemplo, el grupo G4 añadió solo similitudes, pero no hizo ningún cambio en la diferencia errónea inicialmente destacada (se subraya lo añadido tras la puesta en común):

G4: Con respecto a las diferencias, la Ciencia es un conocimiento básico y teórico, mientras que la Tecnología es un conocimiento práctico. [...]Asimismo, la Ciencia busca el avance en la investigación sin necesidad de cubrir mejoras, en cambio, la Tecnología busca satisfacer necesidades sociales. En cuanto a las similitudes, ambas buscan rendimiento económico y manejan conocimientos. (Nivel 1)

Los dos grupos que logran progresar al nivel 3 añaden alguna diferencia o similitud más a su respuesta inicial. Por ejemplo, el grupo G3 se refiere a las publicaciones y a las patentes como principales medios de presentación de los avances científico y tecnológico, respectivamente (se subraya lo añadido a la respuesta inicial):

G3: Diferencias: La Ciencia busca satisfacer la curiosidad y tiene desinterés económico; genera publicaciones; mientras que la Tecnología busca satisfacer necesidades y tiene interés económico; genera patentes. Similitudes: [ambas] están en continuo desarrollo, son experimentales y tratan con conceptos que se pueden cuantificar. (Nivel 3; corchetes añadidos) 
La respuesta final más amplia o completa en esta cuestión correspondió al grupo G2, aunque, tal y como se establece en la rúbrica, no le permitía alcanzar el nivel máximo:

G2: Diferencias: La Ciencia se pregunta “¿Por qué ocurren los fenómenos?”, [mientras que] la Tecnología se pregunta “¿Cómo aplicarlo?”. El principal objetivo de la Ciencia es la producción de conocimiento, que publica (propiedad intelectual). [Y] el principal objetivo es la aplicación del conocimiento, que patenta (explotación). Similitudes: ambas promueven el avance del conocimiento científico y tecnológico, requieren recursos (tiempo, dinero), exigen pensamiento crítico, necesitan puesta en práctica para ser demostradas. Ambas se potencian mutuamente: conocimientos de la Ciencia se aplican en desarrollos tecnológicos y sistemas creados en Tecnología permiten avanzar en el trabajo científico. (Nivel 3; corchetes añadidos)

\section{Conclusiones}

Si bien la investigación presentada es modesta en alcance y desarrollo, supone un avance en la línea de trabajo dedicada a la comprensión de la NDT. Hasta ahora los estudios sobre ello se han limitado básicamente a diagnosticar las concepciones de estudiantes y profesorado sobre NDT (Acevedo-Díaz et al., 2003, 2005; DiGironimo, 2011; García-Carmona, 2006; Ferreira-Gauchía et al., 2011), o hacer planteamientos teóricos con vistas a su introducción en la educación científico-tecnológica (AcevedoDíaz, 1998, 2006; Waight y Abd-El-Khalick, 2012); pero no se han ocupado de implementar y evaluar propuestas educativas específicas para mejorarlas.

A la vista de los resultados obtenidos, se puede decir que, globalmente, los EPFQ lograron una progresión moderada en sus concepciones acerca de los aspectos de NDT tratados. La lectura reflexiva de la controversia entre Tesla y Edison sobre la corriente eléctrica, y la discusión de dos cuestiones de NDT en el contexto de esta, contribuyeron a mejorar algunas de las concepciones iniciales de los EPFQ al respecto; las cuales, en unos casos eran limitadas, y en otros, equivocadas en parte.

De manera más pormenorizada, la evaluación de la experiencia educativa ha revelado fortalezas y debilidades en términos de logros de aprendizaje de los EPFQ sobre los aspectos de NDT tratados. Así, con relación a los componentes que caracterizan la Tecnología como sistema, estos reconocen los instrumentos y artefactos técnicos, habilidades técnicas (know-how), conocimientos científicos, financiación, valores e innovación. En cambio, obvian otros componentes esenciales, tales como: los recursos naturales y artificiales, procesos de producción, control y mantenimiento, factores políticos y las preferencias culturales y estéticas. Unos aspectos de los que prácticamente no se han ocupado los estudios de diagnóstico señalados.

En cuanto a las similitudes y diferencias entre Ciencia y Tecnología, los EPFQ han terminado entendiendo bien que, mientras la Ciencia tiene como fin explicar fenómenos de la naturaleza, la Tecnología se centra en la elaboración de productos (máquinas, artefactos, aplicaciones, etc.) para resolver problemas o necesidades de la Sociedad (Acevedo-Díaz et al., 2003; Raynaud, 2018). También, que los avances de la Ciencia se ponen de manifiesto fundamentalmente en publicaciones y, en cambio, el avance tecnológico se hace palpable con la creación de patentes (Acevedo-Díaz, 1998). Asimismo, queambas disciplinas emplean conocimiento y experimentación, entre otras prácticas, para su desarrollo (Acevedo-Díaz, 2006). Sin embargo, entre algunos 
EPFQ se encuentra cierta resistencia a cambiar la idea ingenua de que la Ciencia es algo "más teórico", y la Tecnología, "más práctica" (Acevedo-Díaz et al., 2003); o bien, que la Ciencia es "más aséptica o independiente de lo social" que la Tecnología.

Por otra parte, la evaluación de la experiencia pone de relieve también la utilidad de actividades como la presentada para favorecer la integración contenidos de NDT en las clases de Física y Química; fundamentalmente, porque suponen intervenciones educativas cortas, que apenas distorsionan el desarrollo del programa previsto para la asignatura. Asimismo, el análisis revela que, aun cuando la lectura de controversias de Historia de la Tecnología es un recurso de un valor pedagógico reseñable, no es per se suficiente para una mejora significativa de las concepciones de los EPFQ sobre un constructo tan poliédrico como NDT. Esto apunta a la necesidad de combinarlo con otros recursos y propuestas para lograr aprendizajes sobre NDT más efectivos. Por ejemplo, mediante la lectura crítica y reflexiva de noticias de la prensa sobre temas tecnocientíficos contemporáneos (Guerrero-Márquez y García-Carmona, 2020). También puede resultar efectivo el planteamiento de proyectos escolares orientados al diseño y construcción de algún artefacto (García-Carmona, 2011), junto con cuestiones para la reflexión explícita sobre aspectos de NDT intervinientes en el proceso (conjugación de Ciencia y Tecnología, aspectos estéticos, utilitarios, etc.). Algo similar se puede hacer con el análisis de objetos tecnológicos cotidianos desde una perspectiva sistémica (García-Carmona y Criado, 2009).

En todo caso, lo esencial será que la NDT sea concebida como un objetivo básico de la alfabetización científico-tecnológica; y partiendo de esta idea, imaginar nuevas estrategias y recursos para su enseñanza/aprendizaje en las clases de Ciencia y Tecnología. Desde esta perspectiva, se asume que lo planteado aquí puede ser igualmente aplicable al área curricular de Tecnología.

Por último, es preciso decir que, dadas las condiciones en las que se llevó a cabo la experiencia educativa, esto es, en un contexto habitual de clase con una muestra de participantes reducida y elegida por conveniencia, los resultados deben acogerse con las limitaciones que ello impone. Sin embargo, estos pueden resultar de interés para animar a otro profesorado de Ciencia y de Tecnología a promover aprendizajes sobre NDT, usando la Historia de la Tecnología. Igualmente pueden servir para motivar nuevos estudios, que profundicen en la comprensión de los aspectos de NDT aquí tratados; o bien, que amplíen la atención a otros aspectos de esta.

\section{Agradecimientos}

Quiero agradecer a mi colega y amigo José Antonio Acevedo Díaz su colaboración en el análisis de los datos del presente estudio.

\section{Bibliografía}

Acevedo-Díaz, J. A. (1998). Análisis de algunos criterios para diferenciar entre Ciencia y Tecnología. Enseñanza de las Ciencias, 16(3), 409-420. https://bit.ly/3jmZLOl

Acevedo-Díaz, J.A. (2006). Modelos de relaciones entre Ciencia y Tecnología: un análisis social e histórico. Revista Eureka sobre Enseñanza y Divulgación de las Ciencias, 3(2), 198-219. https://doi.org/10.25267/Rev_Eureka_ensen_divulg_cienc.2006.v3.i2.03 
Acevedo-Díaz, J. A. (2010). ¿Qué puede aportar la Historia de la Tecnología a la educación CTS? Praxis Pedagógica, 10, 32-39. https://doi.org/10.26620/uniminuto.praxis.10.11.2010.32-39

Acevedo-Díaz, J. A. y García-Carmona, A. (2016). Una controversia de la Historia de la Tecnología para aprender sobre Naturaleza de la Tecnología: Tesla vs. Edison - La guerra de las corrientes. Enseñanza de las Ciencias, 34(1), 193-209. http://dx.doi.org/10.5565/rev/ ensCiencias.1916

Acevedo-Díaz, J. A., Vázquez-Alonso, A., Manassero-Mas, M. A. y Acevedo-Romero, P. (2003). Creencias sobre la Tecnología y sus relaciones con la Ciencia. Revista Electrónica de Enseñanza de las Ciencias, 2(3), 353-376. http://reec.uvigo.es/volumenes/volumen2/ REEC_2_3_9.pdf

Acevedo-Díaz, J. A., Vázquez-Alonso, A., Manassero-Mas, M. A. y Acevedo-Romero, P. (2005). Aplicación de una nueva metodología para evaluar las creencias del profesorado sobre la Tecnología y sus relaciones con la Ciencia. Educación Química, 16(3), 372-382. http:// dx.doi.org/10.22201/fq.18708404e.2005.3.66100

Cajas, F. (2001). Alfabetización científica y tecnológica: la transposición didáctica del conocimiento tecnológico. Enseñanza de las Ciencias, 19(2), 243-254. https://www.raco.cat/index.php/ Ensenanza/article/view/21737

Criado, A. M. y García-Carmona, A. (2011). Investigando las máquinas y artefactos. Sevilla: Díada.

DiGironimo, N. (2011). What is technology? Investigating student conceptions about the nature of technology. International Journal of Science Education, 33(10), 1337-1352. https://doi.or g/10.1080/09500693.2010.495400

Echeverría, J. (2005). La revolución tecnocientífica. CONfines, 1(2), 9-15. https://confines.mty.tesm. $\mathrm{mx} /$ articulos2/EcheverriaJ.pdf

Ferreira-Gauchía, C., Vilches, A. y Gil-Pérez, D. (2012). Concepciones acerca de la Naturaleza de la Tecnología y de las relaciones Ciencia, Tecnología, Sociedad y Ambiente en la educación tecnológica. Enseñanza de las Ciencias, 30(2), 197-218. https://www.raco.cat/index.php/ Ensenanza/article/view/254510

García-Carmona, A. (2006). Concepciones del alumnado de Secundaria sobre las finalidades de la Física y su papel en la Tecnología. Revista Eureka sobre Enseñanza y Divulgación de las Ciencias, 3(2), 188-197. https://revistas.uca.es/index.php/eureka/article/view/3859

García-Carmona, A. (2011). Aprender Física y Química mediante secuencias de enseñanza investigadoras. Archidona (Málaga): Aljibe.

García-Carmona, A. (2020). STEAM, ¿una nueva distracción para la enseñanza de la Ciencia? Ápice. Revista de Educación Científica, 4(2), 35-50. https://doi.org/10.17979/arec.2020.4.2.6533

García-Carmona, A. y Criado, A. M. (2009). ¿Por qué los automóviles son como son? La evolución de un sistema tecnológico. Alambique, 62, 92-106. https://core.ac.uk/download/pdf/51396096.pdf

Guerrero-Márquez, I. y García-Carmona, A. (2020). La energía y su impacto socioambiental en la prensa digital: temáticas y potencialidades didácticas para una educación CTS. Revista Eureka sobre Enseñanza y Divulgación de las Ciencias, 17(3), 3301. https://doi.org/10.25267/ Rev_Eureka_ensen_divulg_cienc.2020.v17.i3.3301

Kline, S.J. (1985). What is technology? Bulletin of Science, Technology, and Society, 5(3), 215-218. https://doi.org/10.1177\%2F027046768500500301

Maiztegui, A., Acevedo, J. A., Caamaño, A., Cañal, P., Carvalho, A. P. M., del Carmen, L. et al. (2002). Papel de la Tecnología en la educación científica: una dimensión olvidada. Revista Iberoamericana de Educación, 28, 129-155. https://doi.org/10.35362/rie280962

Mayring, P. (2000). Qualitative content analysis. Forum: Qualitative Social Research, 1(2), 1-10. https://doi.org/10.17169/fqs-1.2.1089

Ministerio de Educación. (2015). Real Decreto 1105/2014, de 3 de enero, por el que se establece el currículo básico de la Educación Secundaria Obligatoria y del Bachillerato. Madrid: Boletín Oficial del Estado. 
National Research Council. (2012). A framework for K-12 science education: Practices, crosscutting concepts, and core ideas. Washington: The National Academies Press.

Organización para la Cooperación y el Desarrollo Económicos [OCDE]. (2017). Marco de evaluación y de análisis de PISA para el desarrollo: Lectura, matemáticas y ciencias. Versión preliminar. París: OECD Publishing.

Raynaud, D. (2018). ¿Qué es la Tecnología? Pamplona: Laetoli.

Valdés, P., Valdés, R., Guisasola, J. y Santos, T. (2002). Implicaciones de las relaciones CienciaTecnología en la educación científica. Revista Iberoamericana de Educación, 28(1), 101128. https://doi.org/10.35362/rie280961

Waight, N. y Abd-El-Khalick, F. (2012). Nature of Technology: Implications for design, development, and enactment of technological tools in school science classrooms. International Journal of Science Education, 34(18), 2875-2905. https://doi.org/10.1080/09500693.2012.698763

García-Carmona, A. (2021). Reflexiones de estudiantes de profesorado de Física y Química sobre Naturaleza de la Tecnología en el contexto de la controversia Tesla-Edison. Revista Iberoamericana de Educación, 87(1), 35-48. https://doi.org/10.35362/rie8714269 